\title{
On a Model for Quantum Friction III. Ergodic Properties of the Spin - Boson System
}

\author{
Vojkan Jakšić, ${ }^{1,2}$ Claude-Alain Pillet ${ }^{3}$ \\ ${ }^{1}$ Isaac Newton Institute, University of Cambridge, 20 Clarkson Road, Cambridge, CB3 0EH, UK \\ 2 IMA, University of Minnesota, 514 Vincent Hall, 55455-0436 Minneapolis, Minnesota, USA \\ ${ }^{3}$ Département de Physique Théorique, Université de Genève, CH-1211 Genève 4, Switzerland
}

Accepted: 5 October 1995

\begin{abstract}
We investigate the dynamics of a 2-level atom (or spin $\frac{1}{2}$ ) coupled to a mass-less bosonic field at positive temperature. We prove that, at small coupling, the combined quantum system approaches thermal equilibrium. Moreover we establish that this approach is exponentially fast in time. We first reduce the question to a spectral problem for the Liouvillean, a self-adjoint operator naturally associated with the system. To compute this operator, we invoke Tomita-Takesaki theory. Once this is done we use complex deformation techniques to study its spectrum. The corresponding zero temperature model is also reviewed and compared. From a more philosophical point of view our results show that, contrary to the conventional wisdom, quantum dynamics can be simpler at positive than at zero temperature.
\end{abstract}

\section{Introduction}

In this paper we consider the dissipative dynamics of a quantum mechanical 2-level system - the spin - characterized by its two eigenstates of energy $e_{ \pm}= \pm 1$. More specifically we investigate the long time behavior of the dynamics of a spin $\frac{1}{2}$ allowed to interact with a large reservoir. The reservoir is an infinitely extended gas of free, mass-less bosons at positive temperature without Bose-Einstein condensate. We prove that, for sufficiently small coupling, the interacting spin-boson system has strong ergodic properties. In particular it approaches thermal equilibrium exponentially fast. Moreover, the equilibrium state is the unique KMS state of the joint system at the temperature of the heat bath.

The spin-boson system is a simple, yet physically acceptable model for a variety of phenomena related to dissipative quantum tunneling. The literature on the subject is enormous. Let us only mention the review article [LCD] as an excellent introduction to the physical aspects of the model. Also [A1, A2, AM, FNV1, FNV2, FNV3, D1, D2, HS1, HS2, MA, PU, SD, SDLL, RO1, RO2] is a non-exhaustive list of related mathematical investigations. 
The present work is largely based on results previously obtained by the authors in [JP1]. There we have developed perturbative tools suitable for the study of quantum systems with a discrete, possibly infinite, set of energy levels $\left\{e_{i}\right\}$, linearly coupled to a free heat bath at positive temperature. Unfortunately, the general discussion of such systems tends to be technical. For this reason we prefer to restrict ourselves here to a simple model, allowing us to give a more transparent exposition of the underlying ideas. The adaptation of these ideas to more general situations will be presented in a subsequent paper [JP2]. For the interested reader, we also compare the spin-boson model at positive temperature with its zero temperature counterpart. We emphasize that most of the questions answered in this paper are still open problems at zero temperature.

Our argument splits in three conceptually distinct parts: First we formulate an appropriate generalization of Koopman's Lemma to dynamical systems arising from quantum mechanics. This allows us to reduce ergodic properties of the system to spectral problems for a distinguished self-adjoint operator: The Liouvillean. This operator is defined in abstract terms, and we must invoke Tomita-Takesaki's theory to actually compute it. Once the Liouvillean is known, we apply complex deformation techniques to obtain the relevant spectral information. On the technical level, one of the main difficulties is the identification of the Liouvillean. The required results of Tomita-Takesaki theory are readily available in the literature. The final step of the proof boils down to an application of the results in [JP1]. An analysis of resonances reveals the basic mechanism of thermal relaxation. In particular, Einstein's A-B law emerges as Fermi's Golden Rule for the resonances of the Liouvillean.

Acknowledgments. This paper is part of a program suggested to us by I.M. Sigal, to whom we are grateful. We also thank V. Bach, H. Spohn and Ph.A. Martin for useful discussions. V.J. is grateful to J.-P. Eckmann for hospitality at the University of Geneva, where part of this work was done, and Fonds National Suisse for financial support. At an early stage of this work, C.-A.P. was visiting the Institute for Mathematics and its Applications at the University of Minnesota. Its research was also supported by the Fonds National Suisse.

\section{The Model}

In this section we define the spin-boson model. We first introduce the isolated spin and the free reservoir, and discuss their thermal equilibrium states. Recall that a state of a quantum system is a normalized positive linear functional $\mathscr{S}$ on its algebra of observables. A vector state is a state of the form $\mathscr{S}(A)=(\Psi, A \Psi)$, for some unit vector $\Psi$. More generally, a state $\mathscr{S}$ is called normal if there exists a density matrix $\varrho$, a positive trace class operator of unit trace, such that $\mathscr{S}(A)=\operatorname{Tr}(\varrho A)$.

The Hilbert space of the isolated spin is $\mathfrak{H}_{s} \equiv \mathbf{C}^{2}$. Denoting by $\sigma_{x}, \sigma_{y}$ and $\sigma_{z}$ the usual Pauli matrices, we may choose its Hamiltonian to be $H_{s} \equiv \sigma_{z}$. The eigenenergies of the spin are $e_{ \pm}= \pm 1$, and we denote the corresponding eigenstates by $\chi \pm$. Finally, observables of the spin are elements of $M_{2}$, the algebra of all complex $2 \times 2$ matrices. At inverse temperature $\beta$, the equilibrium state of the spin is the normal state defined by the Gibbs Ansatz,

$$
\mathscr{S}_{s}^{\beta}(A) \equiv \frac{1}{Z_{s}^{\beta}} \operatorname{Tr}\left(\exp \left(-\beta H_{s}\right) A\right)
$$


where $Z_{s}^{\beta}$ is a normalization factor. The zero-temperature equilibrium state is obtained in the limit $\beta \uparrow \infty$ : It is the vector state corresponding to the ground state of $H_{s}$,

$$
\mathscr{S}_{s}^{\infty}(A) \equiv(\chi-, A \chi-) .
$$

At vanishing density, the Hilbert space of the free reservoir is the symmetric Fock space constructed over $L^{2}\left(\mathbf{R}^{3}\right)$, which we denote by $\mathfrak{H}_{b}$ (we work in the momentum representation, thus elements of $L^{2}\left(\mathbf{R}^{3}\right)$ are always functions of the momentum $\mathbf{k}$ of an individual boson). Since the bosons are non-interacting, the dynamics of the reservoir is completely determined by the energy $\omega(\mathbf{k})$ of a single boson with momentum $\mathbf{k}$. This dynamics is implemented by the strongly continuous unitary group

$$
\exp \left(i H_{b} t\right) \equiv \Gamma(\exp (i \omega t))
$$

which, by definition, acts on the $N$-particle subspace of $\mathfrak{H}_{b}$ as the $N$-fold tensor product of the one-boson propagator $\exp (i \omega t)$. In terms of the usual creation and annihilation operators $a^{*}(\mathbf{k}), a(\mathbf{k})$, the Hamiltonian $H_{b}$ is given by the familiar formula

$$
H_{b}=d \Gamma(\omega) \equiv \int \omega(\mathbf{k}) a^{*}(\mathbf{k}) a(\mathbf{k}) d^{3} k
$$

In the sequel we restrict ourselves to the physically important case

$$
\omega(\mathbf{k}) \equiv|\mathbf{k}|
$$

However, our method easily accommodates other dispersion laws, as long as the bosons remain mass-less. In this case $H_{b}$ has a simple eigenvalue 0 corresponding to the Fock vacuum $\Omega_{b}$, the remaining part of its spectrum is absolutely continuous and fills the positive real axis. The observables of the reservoir are the field operators

$$
\begin{aligned}
& \varphi(f) \equiv \frac{1}{\sqrt{2}} \int\left(a(\mathbf{k})+a^{*}(\mathbf{k})\right) f(\mathbf{k}) d^{3} k \\
& \pi(f) \equiv \frac{1}{i \sqrt{2}} \int\left(a(\mathbf{k})-a^{*}(\mathbf{k})\right) f(\mathbf{k}) d^{3} k
\end{aligned}
$$

which satisfy the canonical commutation relations (CCR). A mathematically more convenient set of observables is provided by the Weyl system

$$
W(f) \equiv \exp (i \varphi(f))
$$

where $\varphi(f)$ is the self-adjoint (Segal) field operator defined by

$$
\varphi(f) \equiv \frac{1}{\sqrt{2}} \int\left(a(\mathbf{k}) \overline{f(\mathbf{k})}+a^{*}(\mathbf{k}) f(\mathbf{k})\right) d^{3} k
$$

for $f \in L^{2}\left(\mathbf{R}^{3}\right)$. The operators $W(f)$ are unitary on $\mathfrak{H}_{b}$, and satisfy a disguised form of CCR: The Weyl relation

$$
W\left(f_{1}\right) W\left(f_{2}\right)=\exp \left(-i \operatorname{Im}\left(f_{1}, f_{2}\right) / 2\right) W\left(f_{1}+f_{2}\right) .
$$

The dynamics of the reservoir induces a Bogoliubov transformation

$$
\exp \left(i H_{b} t\right) W(f) \exp \left(-i H_{b} t\right)=W(\exp (-i \omega t) f),
$$


of the Weyl system.

It is a well known fact that thermal equilibrium states of extended systems arise in the thermodynamic limit, starting with a system restricted to a finite box $\Lambda \subset \mathbf{R}^{3}$. For such a confined system, the grand canonical ensemble yields a well defined state. Equilibrium states of the extended system are constructed as weak-* limits of these states as $\Lambda \uparrow \mathbf{R}^{3}$. At positive temperature, the equilibrium states obtained in this way have a positive density i.e., an infinite number of particles: They do not fit in the original Fock space (in more technical terms they are not normal). For practical purposes however, it is convenient to restore the familiar Hilbert space framework. This can be achieved by an appropriate choice of the representation of CCR. Let us briefly review some facts about such representations.

Let $\mathfrak{D} \subset L^{2}\left(\mathbf{R}^{3}\right)$ be a dense subspace. Weyl's algebra over $\mathfrak{D}$ is the $\mathrm{C}^{*}$ - algebra generated by the set $\{W(f): f \in \mathfrak{D}\}$. A representation $(\mathscr{H}, \pi)$ of this algebra is called regular if the functions

$$
\mathbf{R} \ni \lambda \mapsto \pi(W(\lambda f))
$$

are strongly continuous for each $f \in \mathfrak{D}$. By Stone's theorem, regularity is equivalent to the existence of a self-adjoint operator $\varphi_{\pi}(f)$ such that

$$
\pi(W(\lambda f))=\exp \left(i \lambda \varphi_{\pi}(f)\right) .
$$

One then refers to the $\varphi_{\pi}(f)$ as the field operators of the representation. A representation is called cyclic if for some $\Omega \in \mathscr{H}$ the set $\{\pi(W(f)) \Omega: f \in \mathfrak{D}\}$ is total in $\mathscr{H}$. To each cyclic representation $(\mathscr{H}, \pi, \Omega)$ of Weyl's algebra, we can associate a generating functional defined by

$$
\mathfrak{D} \ni f \mapsto(\Omega, \pi(W(f)) \Omega) .
$$

Generating functionals of regular, cyclic representations have been characterized by Araki and Segal:

Theorem 2.1. A map $\mathfrak{s}: \mathfrak{D} \rightarrow \mathbf{C}$ is the generating functional of a regular, cyclic representation of Weyl's algebra over $\mathfrak{D}$ if and only if the following conditions are satisfied:

1. $\mathfrak{s}(0)=1$.

2. For each $f \in \mathfrak{D}$ the mapping $\lambda \mapsto \mathfrak{s}(\lambda f)$ is continuous.

3. For each finite subset $\left\{f_{1}, f_{2}, \ldots, f_{n}\right\} \subset \mathfrak{D}$, and any $z_{1}, \ldots, z_{n} \in \mathbf{C}$ one has

$$
\sum_{i, j=1}^{n} \mathfrak{s}\left(f_{i}-f_{j}\right) \exp \left(-i \operatorname{Im}\left(f_{\imath}, f_{j}\right) / 2\right) \bar{z}_{i} z_{j} \geq 0 \text {. }
$$

Furthermore, this representation is unique, up to unitary equivalence.

At zero chemical potential (which is appropriate for mass-less particles), and in the absence of condensate, the thermodynamic limit leads to the following generating functional for the infinite free Bose gas (see for example [BR2, LP or CA])

$$
\mathfrak{s}^{\beta}(f) \equiv \exp \left(-\frac{1}{4} \int(1+2 \rho(\mathbf{k}))|f(\mathbf{k})|^{2} d^{3} k\right),
$$

for $f$ in the dense subspace $\mathfrak{D}_{l o c} \subset L^{2}\left(\mathbf{R}^{3}\right)$ of functions with compactly supported Fourier transform (i.e., localized in the position representation). In Eq. (2.4), the 
function $\rho(\mathbf{k})$ is the equilibrium momentum distribution of the bosons, and is related to their energy according to Planck's radiation law

$$
\rho(\mathbf{k})=\frac{1}{\exp (\beta \omega(\mathbf{k}))-1} .
$$

The energy density of the boson gas is strictly positive, and satisfies the StefanBoltzmann relation

$$
\int \omega(\mathbf{k}) \rho(\mathbf{k}) d^{3} k \propto \beta^{-4} .
$$

Let us denote by $\mathscr{C}_{l o c}$ the Weyl algebra over $\mathfrak{D}_{l o c}$. Since our system has positive density, this is a natural minimal set of observables. By Theorem 2.1, the above functional (2.4) corresponds to a regular, cyclic representation $\left(\mathscr{H}_{b}, \pi_{A W}, \Psi_{b}\right)$ of $\mathscr{C}_{\text {loc }}$. This representation has been explicitly constructed by Araki and Woods (see [AW1, BR2, CH or LP]), and can described as follows: $\mathscr{H}_{b}$ is the space of all HilbertSchmidt operators on $\mathfrak{H}_{b}$ equipped with the inner product

$$
(X, Y) \equiv \operatorname{Tr}\left(X^{*} Y\right) .
$$

The representant of $W(f)$ acts according to

$$
\pi_{A W}(W(f)): X \mapsto W\left((1+\rho)^{1 / 2} f\right) X W\left(\rho^{1 / 2} f\right),
$$

for any $X \in \mathscr{H}_{b}$. Finally the cyclic vector is the projection on the Fock vacuum

$$
\Psi_{b} \equiv \Omega_{b}\left(\Omega_{b}, \cdot\right)
$$

One easily verifies that the state

$$
\mathscr{S}_{b}^{\beta}(A) \equiv\left(\Psi_{b}, \pi_{A W}(A) \Psi_{b}\right),
$$

reproduces the functional (2.4), and that the free dynamics has a unitary implementation in the space $\mathscr{H}_{b}$

$$
\pi_{A W}\left(\exp \left(i H_{b} t\right) A \exp \left(-i H_{b} t\right)\right)=\exp \left(i H_{B} t\right) \pi_{A W}(A) \exp \left(-i H_{B} t\right) .
$$

The generator of this group can be explicitly written as

$$
H_{B}: X \mapsto\left[H_{b}, X\right] .
$$

In the sequel, we will always work in the Araki-Woods representation. Consequently we shall give to the representants $\pi_{A W}(A)$ the status of observables of the boson gas at positive temperature. For reasons which will soon become clear, it is convenient to consider also the von Neumann algebra generated by these representants. Recall that a $\mathrm{C}^{*}$ - algebra of operators on a Hilbert space $\mathfrak{H}$ is a von Neumann algebra if it is closed in the weak operator topology. Let $\mathscr{B}$ be a set of bounded operators on $\mathfrak{H}$. We denote by $\mathscr{B}^{\prime}$ its commutant i.e., the set of bounded operators commuting with all elements of $\mathscr{B}$. If $\mathscr{B}$ is closed under hermitian conjugation, $\mathscr{B}^{\prime}$ is a von Neumann algebra. Moreover the double commutant $\mathscr{B}^{\prime \prime}$ is the smallest von Neumann algebra containing. $\mathscr{P}$ (see [BR1] or [SA]). We define the algebra of observables of the reservoir at positive temperature by

$$
\mathfrak{M}_{b} \equiv \pi_{A W}\left(\mathscr{C}_{l o c}\right)^{\prime \prime} .
$$


Remark 1. The map $\Phi \otimes \bar{\Psi} \mapsto \Phi(\Psi, \cdot)$ provides an isomorphism between $\mathfrak{H}_{b} \otimes \mathfrak{H}_{b}$ and $\mathscr{H}_{b}$. In the sequel we shall identify these two spaces without further mention. For example, the formulae

$$
\begin{gathered}
H_{B}=H_{b} \otimes I-I \otimes H_{b}, \\
\pi_{A W}(W(f))=W\left((1+\rho)^{1 / 2} f\right) \otimes W\left(\rho^{1 / 2} \bar{f}\right),
\end{gathered}
$$

directly follow from Eq. (2.9) under this identification.

In Eq. (2.4), the limit $\beta \uparrow \infty$ yields the generating functional

$$
\mathfrak{s}^{\infty}(f) \equiv \exp \left(-\frac{1}{4} \int_{\mathbf{R}^{3}}|f(\mathbf{k})|^{2} d^{3} k\right)=\left(\Omega_{b}, W(f) \Omega_{b}\right),
$$

which extends to arbitrary $f \in L^{2}\left(\mathbf{R}^{3}\right)$. Thus, as expected, we recover the original Fock space representation and, here again, the zero-temperature equilibrium state is the vector state associated with the ground state of the system. In this limiting case the density of the gas vanishes (see (2.5) and (2.6)), and a natural set of observables is the full Weyl algebra $\mathscr{C}$ over $L^{2}\left(\mathbf{R}^{3}\right)$. Note that this $C^{*}$ - algebra is irreducible i.e., . $\mathscr{A}^{\prime \prime}=\mathscr{B}\left(\mathfrak{H}_{b}\right)$ the space of all bounded linear operators on $\mathfrak{H}_{b}$.

We are now ready to define the spin-boson model. At zero temperature, the Hilbert space of the joint system is

$$
\mathfrak{H} \equiv \mathfrak{H}_{s} \otimes \mathfrak{H}_{b}
$$

and its free Hamiltonian is

$$
\widetilde{H}_{0} \equiv H_{s} \otimes I+I \otimes H_{b}
$$

The coupling of the two subsystems is achieved by addition of an interaction term, namely

$$
\widetilde{H}_{\lambda} \equiv \widetilde{H}_{0}+\lambda Q \otimes \varphi(\alpha)
$$

where $\lambda$ is a real constant, $Q \equiv \sigma_{x}$, and $\alpha \in L^{2}\left(\mathbf{R}^{3}\right)$. If

$$
\left(1+\omega^{-1 / 2}\right) \alpha \in L^{2}\left(\mathbf{R}^{3}\right),
$$

then, by standard estimates (see [GJ], Sect. 1.2), the interaction term $Q \otimes \varphi(\alpha)$ is infinitesimally small with respect to $\widetilde{H}_{0}$. Thus the operator defined by Eq. (2.11) is essentially self-adjoint on $\mathfrak{H}_{s} \otimes D\left(H_{b}\right)$. For simplicity we will also denote by $\widetilde{H}_{\lambda}$ its self-adjoint extension. The dynamics of the model is given by

$$
\tilde{\tau}_{\lambda}^{t}: A \mapsto \exp \left(i \widetilde{H}_{\lambda} t\right) A \exp \left(-i \widetilde{H}_{\lambda} t\right)
$$

Generally, the algebra $M_{2} \otimes \mathscr{C}$ is not invariant under $\tilde{\tau}_{\lambda}$. From the analytical point of view, the situation is even worse since $\tilde{\tau}_{\lambda}$ is not continuous in the natural topology of this algebra. To obtain a decent dynamics we must extend the set of observables to the enveloping von Neumann algebra which, by the last remark of the previous paragraph, is $\mathscr{B}(\mathfrak{H})$. Since the function $t \mapsto \tilde{\tau}_{\lambda}^{t}(A)$ is continuous in the weak operator topology, Hypothesis (2.12) ensures that the spin-boson model defines a $\mathrm{W}^{*}$ - dynamical system $\left(\mathscr{B}(\mathfrak{H}), \tilde{\tau}_{\lambda}\right)$ for any $\lambda \in \mathbf{R}$. Under the stronger condition

$$
\left(1+\omega^{-1}\right) \alpha \in L^{2}\left(\mathbf{R}^{3}\right),
$$


the spectrum of the spin-boson Hamiltonian (2.11) is given by

$$
\sigma\left(\widetilde{H}_{\lambda}\right)=\left[e_{-}(\lambda), \infty[\right.
$$

and $e_{-}(\lambda)$ is a simple eigenvalue (see e.g. [SP]). We denote the associated normalized eigenvector by $\Psi_{\lambda}$. The equilibrium state of the spin-boson system at zero temperature is, by definition, the vector-state defined by

$$
\mathscr{S}_{\lambda}^{\infty}(A) \equiv\left(\Psi_{\lambda}, A \Psi_{\lambda}\right)
$$

Although very natural, this definition is ultimately justified by the fact that, on $M_{2} \otimes$ $\mathscr{C}_{\text {loc }}$, the state $\mathscr{S}_{\lambda}{ }^{\infty}$ is the weak*-limit as $\beta \uparrow \infty$, of the thermal equilibrium states $\mathscr{S}_{\lambda}^{\beta}$ to be defined below (see [SP]).

At positive temperature, the Hilbert space of the joint system is $\mathfrak{H}_{s} \otimes \mathscr{H}_{b}$, and its free dynamics is generated by the Hamiltonian

$$
H_{0} \equiv H_{s} \otimes I+I \otimes H_{B} .
$$

Denoting by $\varphi_{A W}(f)$ the field operators of the Araki-Woods representation, we define the Hamiltonian of the interacting system by

$$
H_{\lambda} \equiv H_{0}+\lambda Q \otimes \varphi_{A W}(\alpha) .
$$

From the physical point of view, this is just a rephrasing of Definition (2.11) in a different representation. However, in a more mathematical perspective, the existence of an intertwining relation of the type (2.8) between (2.11) and (2.15) is a difficult question which, in our opinion, requires more information on the thermodynamic limit $\Lambda \uparrow \mathbf{R}^{3}$ (see for example the discussion in Sect. 5.2.5 of [BR2] or Sect. V.1.4 of [HA]). Since this problem is of little physical relevance we will not pay more attention to it and accept (2.15) as a definition of the model at positive temperature.

In [JP1] we proved that $H_{\lambda}$ is essentially self-adjoint on $\mathfrak{H}_{s} \otimes D\left(H_{b}\right) \otimes D\left(H_{b}\right)$ for any $\lambda \in \mathbf{R}$, provided

$$
\left(\omega+\omega^{-1}\right) \alpha \in L^{2}\left(\mathbf{R}^{3}\right) .
$$

Again we shall use the same symbol to denote its self-adjoint extension. Note that in this case the interaction $Q \otimes \varphi_{A W}(\alpha)$ is not $H_{0}$-bounded. Under the above assumption, it is well known that

$$
\tau_{\lambda}^{t}: A \mapsto \exp \left(i H_{\lambda} t\right) A \exp \left(-i H_{\lambda} t\right)
$$

maps the von Neumann algebra

$$
\mathfrak{M} \equiv M_{2} \otimes \mathfrak{M}_{b}=\left(M_{2} \otimes \pi_{A W}\left(\mathscr{C}_{l o c}\right)\right)^{\prime \prime},
$$

into itself (see [FNV1] or [SP] for example, but also Sect. 6). Since on the other hand the function $t \mapsto \tau_{\lambda}^{t}(A)$ is weakly continuous (in fact it is continuous in the $\sigma$-strong* topology), the spin-boson model at positive temperature also defines a $\mathrm{W}^{*}$ - dynamical system $\left(\mathfrak{M}, \tau_{\lambda}\right)$. Thermal equilibrium states of such systems are characterized by the KMS condition.

Definition 2.2. Let $(\mathfrak{N}, \tau)$ be a $\mathrm{W}^{*}$ - dynamical system, and $\beta>0$. A state $\mathscr{S}$ on $\mathfrak{N}$ is a $(\tau, \beta)-K M S$ state if it satisfies:

1. $\mathscr{S}$ is normal. 
2. For any $A, B \in \mathfrak{N}$ there exists a function $F_{A, B}(z)$, analytic in the strip

$$
\{z: 0<\operatorname{Im}(z)<\beta\},
$$

continuous on its closure, and satisfying the KMS boundary conditions

$$
\begin{aligned}
F_{A, B}(t) & =\mathscr{S}\left(A \tau^{t}(B)\right), \\
F_{A, B}(t+i \beta) & =\mathscr{S}\left(\tau^{t}(B) A\right),
\end{aligned}
$$

for $t \in \mathbf{R}$.

The following well known fact, first proved in [FNV1], will naturally follow from our discussion in Sect. 6 (see Theorem 6.1).

Proposition 2.3. For any $\lambda \in \mathbf{R}$ and $\beta>0$, there exists a unique $\left(\tau_{\lambda}, \beta\right)$-KMS state $S_{\lambda}^{\beta}$ on $\mathfrak{M}$.

Remark 2. In the sequel we refer to $\lambda$ as the friction constant, and to $\alpha$ as the form factor.

\section{Results}

According to the previous section we shall, from now on, assume that

(H1) The form factor $\alpha$ in Eq. (2.11) and (2.15) satisfies

$$
\left(\omega+\omega^{-1}\right) \alpha \in L^{2}\left(\mathbf{R}^{3}\right) .
$$

For technical reasons related to the use of complex deformation techniques in [JP1], we also need a regularity assumption on the form factor $\alpha$. To state this hypothesis we need some additional notation. If $\mathfrak{h}$ is a Hilbert space, we denote by $H^{2}(\delta, \mathfrak{h})$ the Hardy class of $\mathfrak{h}$-valued functions on the strip

$$
\mathfrak{S}(\delta) \equiv\{z:|\operatorname{Im}(z)|<\delta\} .
$$

The Hilbert space $H^{2}(\delta, \mathfrak{h})$ consists of all analytic functions $f: \mathfrak{S}(\delta) \rightarrow \mathfrak{h}$ satisfying

$$
\|f\|_{H^{2}(\delta, \mathfrak{h})} \equiv \sup _{|a|<\delta} \int_{-\infty}^{\infty}\|f(x+i a)\|_{\mathfrak{h}}^{2} d x<\infty .
$$

Let $S^{2}$ denote the unit sphere in $\mathbf{R}^{3}$. Given a function $f$ on $\mathbf{R}^{3}$, we define a new function $\tilde{f}$ on $\mathbf{R} \times \mathrm{S}^{2}$ by the formula

$$
\tilde{f}(s, \hat{k}) \equiv\left\{\begin{array}{cc}
-|s|^{1 / 2} \bar{f}(|s| \hat{k}) & \text { if } s<0 \\
s^{1 / 2} f(s \hat{k}) & \text { if } s \geq 0
\end{array}\right.
$$

Our central technical hypothesis is:

(H2) There exists $0<\delta<2 \pi / \beta$ such that

$$
\tilde{\alpha} \in H^{2}\left(\delta, L^{2}\left(\mathrm{~S}^{2}\right)\right) \text {. }
$$


Finally we must assume that the spin effectively couples to the reservoir at Bohr's frequency $\Delta \omega=\left|e_{+}-e_{-}\right|=2$,

(H3) $\int_{\mathrm{S}^{2}}|\alpha(2 \hat{k})|^{2} d \sigma(\hat{k})>0$

where $d \sigma$ is the surface measure on $\mathrm{S}^{2}$.

The most severe restriction on the infrared behavior of $\alpha$ is Condition (H1) which requires $\alpha(\mathbf{k})=O\left(|\mathbf{k}|^{\eta}\right)$ as $\mathbf{k} \rightarrow 0$ with $\eta>-1 / 2$. Conditions (H1)-(H3) are satisfied, for example, by the function $\alpha(\mathbf{k})=\sqrt{|\mathbf{k}|} \exp \left(-|\mathbf{k}|^{2}\right)$. More general conditions will be discussed in [JP2]. We are now ready to formulate the problem of return to equilibrium. Our discussion is motivated by the work of Robinson ([RO1, RO2]).

Definition 3.1. The spin-boson system at zero temperature has the property of return to equilibrium if

$$
\lim _{|t| \rightarrow \infty} \mathscr{S}\left(\tilde{\tau}_{\lambda}^{t}(A)\right)=\mathscr{S}_{\lambda}^{\infty}(A)
$$

for any normal state $\mathscr{S}$ and any $A \in M_{2} \otimes \mathscr{C}_{\text {loc }}$

Definition 3.2. The spin-boson system at positive temperature has the property of return to equilibrium if

$$
\lim _{|t| \rightarrow \infty} \mathscr{S}\left(\tau_{\lambda}^{t}(A)\right)=\mathscr{S}_{\lambda}^{\beta}(A),
$$

for any normal state $\mathscr{S}$ and $A \in \mathfrak{M}$.

We remark that, whenever Relation (3.2) or (3.3) holds, one would also like to know the rate at which the limit is achieved.

In the zero-temperature case, the question of return to equilibrium is still an open and, we believe, an outstanding problem of mathematical physics. We will briefly discuss this problem and related difficulties below. For additional information we refer the reader to [HE, SC and HS1].

Let us now state the main results of this paper which, in view of the difficulties encountered in the zero-temperature case, come perhaps as a surprise.

Theorem 3.3. Suppose that Hypotheses (H1)-(H3) hold. Then, for $\beta>0$, there exists a constant $\ell(\beta)>0$, depending only on the form factor $\alpha$, and such that the spin-boson system has the property of return to equilibrium for any real $\lambda$ satisfying $0<|\lambda|<\ell(\beta)$.

Remark 1. An immediate consequence of this theorem is that, for any density matrix $\rho$ on the space $\mathfrak{H}_{s} \otimes \mathscr{H}_{b}$ and any $X \in M_{2}$, we have

$$
\lim _{\lambda \rightarrow 0} \lim _{t \rightarrow \infty} \operatorname{Tr}\left(\rho \tau_{\lambda}^{t}(X \otimes I)\right)=\frac{\operatorname{Tr}\left(\exp \left(-\beta H_{s}\right) X\right)}{\operatorname{Tr}\left(\exp \left(-\beta H_{s}\right)\right)} .
$$

For a related discussion in the framework of master equations, see [D1 and D3], Eq. 5.13.

Remark 2. We can recover the zero-temperature model in the limit $\beta \uparrow \infty$. Indeed, the partial trace of any normal state $\mathscr{S}$ of the positive temperature model over the third space in $\mathfrak{H}_{s} \otimes \mathfrak{H}_{b} \otimes \mathfrak{H}_{b}$ is a normal state $\widetilde{\mathscr{S}}$ of the zero temperature model. In 
fact any such state can be obtained in this way. Moreover one can show that for any normal state $\mathscr{S}$,

$$
\begin{gathered}
\lim _{\beta \uparrow \infty} \mathscr{T}_{\lambda}^{\beta}\left(X \otimes \pi_{A W}(W(f))\right)=\mathscr{S}_{\lambda}^{\infty}(X \otimes W(f)), \\
\lim _{\beta \uparrow \infty} \mathscr{S}\left(\tau_{\lambda}^{t}\left(X \otimes \pi_{A W}(W(f))\right)\right)=\widetilde{\mathscr{S}}\left(\tilde{\tau}_{\lambda}^{t}(X \otimes W(f))\right),
\end{gathered}
$$

hold for arbitrary $X \in M_{2}$ and $f \in \mathfrak{D}_{\text {loc }}$. However this limit is quite singular and, by the best estimate we have, the constant of Theorem 3.3 vanishes like $\ell(\beta)=O\left(\beta^{-1}\right)$ as $\beta \uparrow \infty$. Thus, our result does not yield any new information concerning the zerotemperature spin-boson system.

Theorem 3.4. Suppose that Hypotheses (H1)-(H3) hold and let $\ell(\beta)$ be as in Theorem 3.3. There exist a norm dense set of normal states $\mathscr{N}_{0}$ and a strongly dense $*$-algebra $\mathfrak{M}_{0} \subset \mathfrak{M}$, both independent of $\beta$, and such that for $|\lambda|<\ell(\beta), \mathscr{S} \in \mathscr{N}_{0}$ and $A \in \mathfrak{M}_{0}$, one has

$$
\left|\mathscr{S}\left(\tau_{\lambda}^{t}(A)\right)-\mathscr{T}_{\lambda}^{\beta}(A)\right| \leq C(\mathscr{S}, A) \mathrm{e}^{-\gamma(\lambda)|t|},
$$

for some $C(\mathscr{S}, A)$ independent of $\lambda$ and $\beta$. The function $\gamma(\lambda)$ is strictly positive for $0<|\lambda|<\ell(\beta)$, and satisfies

$$
\gamma(\lambda)=\lambda^{2} \frac{4 \pi}{\operatorname{th} \beta} \int_{\mathrm{S}^{2}}|\alpha(2 \hat{k})|^{2} d \sigma(\hat{k})+O\left(\lambda^{4}\right)
$$

as $\lambda \rightarrow 0$.

Remark 3. $\quad \gamma(\lambda)$ is the negative imaginary part of the complex resonance of the Liouvillean which is closest to the real axis (see Sect. 6 for details).

Remark 4. For any $X \in M_{2}$, we have $X \otimes I \in \mathfrak{M}_{0}$.

The proofs will be presented in Sect. 6. We now turn to the promised discussion of the mechanisms behind thermal relaxation. To emphasize the physical content of the model, we shall use its atom-photon interpretation (see e.g., [CDG]). We discuss first the zero-temperature case. The relevant physical process is radiative decay via spontaneous emission. The atom "radiates" its energy into the vacuum. As the emitted photons propagate towards infinity, the interacting system dissipates to its lowest energy state: The ground state. We discuss the spectral consequences of this process in some detail in the sequel.

The spectrum of the uncoupled Hamiltonian $\widetilde{H}_{0}$ is given by

$$
\begin{aligned}
& \sigma_{a c}\left(\widetilde{H}_{0}\right)=[-1, \infty[, \\
& \sigma_{s c}\left(\widetilde{H}_{0}\right)=\emptyset, \\
& \sigma_{p p}\left(\widetilde{H}_{0}\right)=\{-1,+1\} .
\end{aligned}
$$

As the coupling is "switched on," the lowest eigenvalue moves along the real axis to a new location $e_{-}(\lambda)$. It remains simple, merely experiencing what is called the Lamb shift. The fate of the other eigenvalue is different: Since it is embedded in the continuous spectrum we expect it to turn into a resonance, in some appropriate sense. This scenario is still a conjecture, nevertheless there exists a well developed 
formal method, going under the name time-dependent perturbation theory, which has been used since the 20's to compute the coefficients in the formal Taylor expansion of $e_{+}(\lambda)$ (see [DI, HE and SC]). The imaginary part of the first non-trivial term in this expansion is generally known as Fermi's Golden Rule. For the model (2.11), this method yields

$$
\operatorname{Im}\left(e_{+}(\lambda)\right)=-\lambda^{2} 4 \pi \int_{\mathrm{S}^{2}}|\alpha(2 \hat{k})|^{2} d \sigma(\hat{k})+O\left(\lambda^{4}\right) .
$$

To second order, this quantity is the probability per unit time for the atom to spontaneously emit a photon while making a transition $\chi_{+} \rightarrow \chi-$ (see [WW and HE] for more details).

If the above picture is not qualitatively altered by re-absorption and other higher order processes, we expect the following spectrum

$$
\begin{aligned}
\sigma_{a c}\left(\widetilde{H}_{\lambda}\right) & =\left[e_{-}(\lambda), \infty[,\right. \\
\sigma_{s c}\left(\widetilde{H}_{\lambda}\right) & =\emptyset, \\
\sigma_{p p}\left(\widetilde{H}_{\lambda}\right) & =\left\{e_{-}(\lambda)\right\},
\end{aligned}
$$

the eigenvalue $e_{-}(\lambda)$ being simple. From this and the fact that

$$
\mathscr{S}_{\lambda}^{\infty}\left(A \tilde{\tau}_{\lambda}^{t}(B)\right)=\left(A^{*} \Psi_{\lambda}, \exp \left(i\left(\widetilde{H}_{\lambda}-e_{-}(\lambda)\right) t\right) B \Psi_{\lambda}\right),
$$

an elementary property of absolutely continuous spectrum ([RS], Sect. 3, Lemma 2), would imply the mixing property of the spin-boson system at zero-temperature:

$$
\lim _{|t| \rightarrow \infty} \mathscr{S}_{\lambda}^{\infty}\left(A \tilde{\tau}_{\lambda}^{t}(B)\right)=\mathscr{S}_{\lambda}^{\infty}(A) \mathscr{S}_{\lambda}^{\infty}(B)
$$

for any observables $A, B \in M_{2} \otimes$. Cloc $_{\text {loc }}$ One easily shows that (3.2) implies (3.6), but the opposite is not true: At zero-temperature, mixing is strictly weaker than return to equilibrium. We shall see in Sect. 4 that the situation is different at positive temperature.

In our opinion, the first step towards a proof of return to equilibrium at zero temperature should be a derivation of (3.5), or at least of the mixing property (3.6). However, in view of the previous discussion, we believe that there is no natural way to map this question into a purely spectral problem, and that a new idea is needed.

Important advances in this direction have been recently made in [HS1, HS2]. In the first paper, Hübner and Spohn develop a scattering theory for the model. In particular, they show that return to equilibrium is a consequence of asymptotic completeness. The second paper presents an adaptation of Mourre theory to the massive spin-boson model at zero temperature. It would be very interesting to extend these results to the mass-less model discussed here.

When this work was finished, we learned that Bach, Frölich and Sigal announced a result which, specialized to the model (2.11), yields (3.5) under some technical assumptions on $\alpha$, e.g. dilation analyticity ([BFS]). In addition, their results in essence justify the resonance picture sketched above.

In the remaining part of this section we briefly discuss the situation at positive temperature. Here two elementary processes are responsible for thermal relaxation: Absorption and induced emission. The atom continuously exchanges energy with the 
radiation field. The resulting thermal fluctuations drive the system towards equilibrium. The operator $H_{0}$ has the following spectrum:

$$
\begin{aligned}
& \sigma_{a c}\left(H_{0}\right)=\mathbf{R}, \\
& \sigma_{s c}\left(H_{0}\right)=\emptyset, \\
& \sigma_{p p}\left(H_{0}\right)=\{-1,+1\} .
\end{aligned}
$$

Unlike in the zero-temperature case, we expect here both eigenvalues to turn into resonances as a result of the coupling. This was rigorously established by the authors in [JP1], where Theorem 2.2 translates into the following statement.

Theorem 3.5. Suppose that Hypotheses (H1)-(H3) hold. Then there exists a dense subspace $\mathscr{E} \subset \mathfrak{H}_{s} \otimes \mathscr{H}_{b}$ and, for $0<\eta<\delta$, a constant $\Lambda(\eta)>0$ such that if $|\lambda|<\Lambda(\eta)$ and $\Phi, \Psi \in \mathscr{E}$, the functions

$$
z \mapsto\left(\Phi,\left(H_{\lambda}-z\right)^{-1} \Psi\right)
$$

have a meromorphic continuation from the upper half-plane onto the region

$$
\text { (?) } \equiv\{z: \operatorname{Im}(z)>-\eta\} \text {. }
$$

On $\odot$, the functions (3.7) are analytic except for two simple poles located at $E_{ \pm}(\lambda)$. The functions $E_{ \pm}(\lambda)$ are analytic for $|\lambda|<\Lambda(\eta)$. Furthermore the first coefficient in the Taylor expansion

$$
E_{ \pm}(\lambda)= \pm 1+a_{ \pm}^{(2)} \lambda^{2}+\ldots
$$

is given by

$$
\begin{gathered}
\Gamma_{ \pm}^{\beta} \equiv-\operatorname{Im}\left(a_{ \pm}^{(2)}\right)=2 \pi \frac{\exp ( \pm \beta)}{|\operatorname{sh}(\beta)|} \int_{\mathrm{S}^{2}}|\alpha(2 \hat{k})|^{2} d \sigma(\hat{k}) \\
\Pi_{ \pm}^{\beta} \equiv \operatorname{Re}\left(a_{ \pm}^{(2)}\right)= \pm \frac{1}{2} \operatorname{PV} \int_{\mathbf{R} \times \mathrm{S}^{2}} \frac{\exp ( \pm \beta s / 2)}{|\operatorname{sh}(\beta s / 2)|} \frac{|\tilde{\alpha}(s, \hat{k})|^{2}}{2-s} d s d \sigma(\hat{k}),
\end{gathered}
$$

where PV stands for Cauchy's principal value.

As an immediate consequence we obtain that, for small non-zero $\lambda$,

$$
\begin{aligned}
\sigma_{a c}\left(H_{\lambda}\right) & =\mathbf{R}, \\
\sigma_{s c}\left(H_{\lambda}\right) & =\emptyset, \\
\sigma_{p p}\left(H_{\lambda}\right) & =\emptyset .
\end{aligned}
$$

Moreover we remark that the imaginary part of $E_{ \pm}(\lambda)$ is related to the radiative lifetime of the corresponding atomic state $\chi \pm$. In second order perturbation theory, only processes in which a single photon is either emitted or absorbed are taken into account. In this framework, the coefficients $\Gamma_{ \pm}^{\beta}$ are related to Einstein's A-B law ([E], see also [PA and P]). We refer to [JP1] for a detailed discussion.

The spectral analysis of $H_{\lambda}$ is only a first step toward the understanding of the long time behavior of the spin-boson model, and the proof of (3.3) requires a new ingredient. In the next section, we show how to exploit the rich algebraic structure of the positive temperature model to reformulate the question of return to equilibrium as a spectral problem. 


\section{Quantum Koopmanism}

The spectral approach to classical dynamics is based on Koopman's lemma: Let $(M, \mu, \varphi)$ be a classical dynamical system i.e., $M$ a measure space, $\mu$ a probability measure on $M$ and $t \mapsto \varphi^{t}$ a measurable group of measure preserving transformations of $M$. If $L^{2}(M, d \mu)$ is separable, then $f \mapsto f \circ \varphi^{t}$ defines a strongly continuous unitary group with generator $L$. The ergodic properties of the system can be characterized by the spectrum of $L$ (see [CFS] for more details). Note that if the system is Hamiltonian, $L$ is nothing but the familiar Liouville operator.

Our aim is to extend Koopman's framework to quantum mechanics. Let $\mathfrak{M}$ be a von Neumann algebra. Recall that a state $\mathscr{S}$ on $\mathfrak{M}$ is faithful if $\mathscr{S}\left(A^{*} A\right)=0$ implies $A=0$. We shall call $(\mathfrak{M}, \mathscr{S}, \tau)$ a quantum dynamical system if $t \mapsto \tau^{t}$ is a weakly continuous group of automorphisms of $\mathfrak{M}$, and $\mathscr{S}$ a faithful normal $\tau$-invariant state. We further denote by $(\mathscr{H}, \pi, \Omega)$ the canonical cyclic representation of $\mathfrak{M}$ (see [BR1], Sect. 2.3.3) associated to $\mathscr{S}$. The two conditions

$$
\begin{aligned}
\pi\left(\tau^{t}(A)\right) & =\exp (i L t) \pi(A) \exp (-i L t), \\
L \Omega & =0,
\end{aligned}
$$

uniquely determine a self-adjoint operator $L$ on $\mathscr{H}$. In analogy with the classical case, we call $L$ the Liouvillean of the system (see [RO1], where $L$ is called equilibrium Hamiltonian). Note that the second condition in (4.1) is crucial: Many operators satisfy the first condition. In fact if $L_{0}$ is such an operator, so is $L_{0}+V$ for any self-adjoint $V \in \pi(\mathfrak{M})^{\prime}$.

It is a simple exercise to show that if $(\mathfrak{M}, \mathscr{S}, \tau)$ is the $\mathrm{W}^{*}$-dynamical system naturally associated to $(M, \mu, \varphi)$, the Liouvillean reduces to the familiar Koopman operator. We will show that the spectral characterizations of ergodic properties carry over to the quantum case. To formulate our result, we need the following definitions.

Definition 4.1. Let $(\mathfrak{M}, \mathscr{S}, \tau)$ be a quantum dynamical system, and denote by $\mathscr{N}$ the set of normal states on $\mathfrak{M}$.

1. $(\mathfrak{M}, \mathscr{S}, \tau)$ is ergodic if, for any $A \in \mathfrak{M}$ and $\mathscr{S}^{\prime} \in \mathscr{N}$, one has

$$
\lim _{T \rightarrow \infty} \frac{1}{2 T} \int_{-T}^{T} \mathscr{S}^{\prime}\left(\tau^{t}(A)\right) d t=\mathscr{S}(A) .
$$

2. It is weakly mixing if, for any $A, B \in \mathfrak{M}$, one has

$$
\lim _{T \rightarrow \infty} \frac{1}{2 T} \int_{-T}^{T}\left|\mathscr{S}\left(\tau^{t}(A) B\right)-\mathscr{S}(A) \mathscr{S}(B)\right|^{2} d t=0 .
$$

3. It is mixing if, for any $A, B \in \mathfrak{M}$, one has

$$
\lim _{t \rightarrow \infty} \mathscr{S}\left(\tau^{t}(A) B\right)=\mathscr{S}(A) \mathscr{S}(B) .
$$

4. It returns to equilibrium if, for any $A \in \mathfrak{M}$ and $\mathscr{S}^{\prime} \in \mathscr{N}$, one has

$$
\lim _{t \rightarrow \infty} \mathscr{S}^{\prime}\left(\tau^{t}(A)\right)=\mathscr{S}(A) .
$$


Theorem 4.2. Let $(\mathfrak{M}, \mathscr{S}, \tau)$ be a quantum dynamical system, $(\mathscr{H}, \pi, \Omega)$ its cyclic representation and $L$ its Liouvillean. Denote also by $P_{\Omega}$ the orthogonal projection of $\mathscr{H}$ along the cyclic vector $\Omega$. Then,

1. $(\mathfrak{M}, \mathscr{S}, \tau)$ is ergodic if and only if 0 is a simple eigenvalue of $L$.

2. It is weakly mixing if and only if $L$ has purely continuous spectrum, except for the simple eigenvalue 0.

3. It is mixing if and only if

$$
\mathrm{w}-\lim _{t \rightarrow \infty} \exp (-i L t)=P_{\Omega} .
$$

\section{It returns to equilibrium if and only if it is mixing.}

Proof. We start with some basic facts (see [BR1], Sect. 2.3.1, 2.4.4 and 2.5.1). Let $\mathfrak{N}$ be a von Neumann algebra on a Hilbert space $\mathfrak{H}$. A vector $\Psi \in \mathfrak{H}$ is called cyclic for $\mathfrak{N}$ if $\mathfrak{N} \Psi$ is dense in $\mathfrak{H}$. It is called separating for $\mathfrak{N}$ if $A \in \mathfrak{N}$ and $A \Psi=0$ implies $A=0$. A vector is separating for $\mathfrak{N}$ if and only if it is cyclic for $\mathfrak{N}^{\prime}$. A representation $\eta$ of $\mathfrak{N}$ is called faithful if it is a $*$-isomorphism between $\mathfrak{N}$ and $\eta(\mathfrak{N})$. A representation $\eta$ is faithful if and only if $\eta(A)=0$ implies $A=0$. A representation is faithful if and only if it satisfies $\|\eta(A)\|=\|A\|$ for any $A \in \mathfrak{N}$. Finally if $\eta$ is a faithful representation of $\mathfrak{N}$ in the Hilbert space $\mathscr{H}$, then a state $\mathscr{S}$ on $\mathfrak{N}$ is normal if and only if there exists a density matrix $\rho$ on $\mathscr{H}$ such that $\mathscr{S}(A)=\operatorname{Tr}(\rho \eta(A))$.

The proof of Theorem 4.2 is based on the following argument: The cyclic representation $\pi$ inherits the faithfulness of $\mathscr{S}$, therefore $\Omega$ is not only cyclic but also separating for $\pi(\mathfrak{M})$. It follows that both $\pi(\mathfrak{M}) \Omega$ and $\pi(\mathfrak{M})^{\prime} \Omega$ are dense in $\mathscr{H}$. Let us denote by $\mathscr{N}_{0}$ the set of vector states arising from vectors in $\pi(\mathfrak{M})^{\prime} \Omega$. The set of finite convex linear combinations of elements of $\mathscr{N}_{0}$ is norm dense in $\mathscr{N}$, thus we can replace $\mathscr{N}$ by $\mathscr{N}_{0}$ in Definition 4.1. Now, for $\mathscr{S}^{\prime} \in \mathscr{N}_{0}$, there exists an operator $C \in \pi(\mathfrak{M})^{\prime}$ such that

$$
\begin{aligned}
\mathscr{S}^{\prime}\left(\tau^{t}(A)\right)-\mathscr{S}(A) & =\left(C \Omega, \pi\left(\tau^{t}(A)\right) C \Omega\right)-(\Omega, \pi(A) \Omega) \\
& =\left(\Omega, \pi\left(\tau^{t}(A)\right) C^{*} C \Omega\right)-(\Omega, \pi(A) \Omega)\left(\Omega, C^{*} C \Omega\right) \\
& =\left(\pi\left(A^{*}\right) \Omega, \exp (-i L t)\left(I-P_{\Omega}\right) C^{*} C \Omega\right),
\end{aligned}
$$

for any $A \in \mathfrak{M}$. In the same way we can write

$$
\begin{aligned}
\mathscr{S}\left(\tau^{t}(A) B\right)-\mathscr{S}(A) \mathscr{S}(B) & =\left(\Omega, \pi\left(\tau^{t}(A) B\right) \Omega\right)-(\Omega, \pi(A) \Omega)(\Omega, \pi(B) \Omega) \\
& =\left(\pi\left(A^{*}\right) \Omega, \exp (-i L t)\left(I-P_{\Omega}\right) \pi(B) \Omega\right),
\end{aligned}
$$

for any $A, B \in \mathfrak{M}$. Von Neumann's ergodic theorem (see [CFS] for example) applied to (4.3) and the density of $\pi(\mathfrak{M}) \Omega$ and $\pi(\mathfrak{M})^{\prime} \Omega$ yield a proof of (i). In a completely similar way, RAGE theorem (see [RS], Theorem XI.115) applied to (4.4) and the density of $\pi(\mathfrak{M}) \Omega$ prove (ii). By (4.4), assertion (iii) is an immediate consequence of the density of $\pi(\mathfrak{M}) \Omega$. Finally, using (iii), we reduce the proof of assertion (iv) to the equivalence of return to equilibrium with (4.2). This follows directly from (4.3), the density of $\pi(\mathfrak{M}) \Omega$ and $\pi(\mathfrak{M})^{\prime} \Omega$, and the fact that an arbitrary $P \in \pi(\mathfrak{M})^{\prime}$ is a linear combination of positive operators.

As in the classical case, one has the

Corollary 4.3. If the Liouvillean $L$ of a quantum dynamical system has purely absolutely continuous spectrum, except for the simple eigenvalue 0 , then this system is mixing. 
Remark 1. Let $(\mathfrak{M}, \tau)$ be a W*-dynamical system. Any $(\tau, \beta)$-KMS state $\mathscr{S}^{\beta}$ is faithful, normal and $\tau$-invariant (see [BR2], Sect. 5.3.1). It follows that $\left(\mathfrak{M}, \mathscr{S}^{\beta}, \tau\right)$ is a quantum dynamical system. This contrasts with the zero temperature case: At zero temperature an equilibrium state (ground state) generally fails to be faithful. The loss of faithfulness in the limit $\beta \uparrow \infty$ is the source of one of the previously mentioned difficulties in the zero temperature spin-boson model: There is no Koopman Lemma at zero temperature, hence no spectral characterization of the dynamics. This is not a quantum phenomenon, the problem already exists in the classical description.

\section{Modular Theory}

In this section we restrict ourselves to quantum dynamical systems of the form $\left(\mathfrak{M}, \mathscr{S}^{\beta}, \tau\right)$, where $\mathscr{S}^{\beta}$ is a $(\tau, \beta)$-KMS state. We show how Tomita-Takesaki's theory relates the Liouvillean of the system to its modular structure, and how this fact naturally leads to multiplicative and additive perturbation theory of the Liouvillean. We start with a brief review of the basic construction leading to modular theory. For a more detailed exposition from the standpoint of mathematical physics we refer the reader to [AR1 and BR1,BR2].

Let $\mathfrak{N}$ be a von Neumann algebra on a Hilbert space $\mathfrak{H}$, and $\Psi \in \mathfrak{H}$ a separating cyclic vector. The map

$$
A \Psi \mapsto A^{*} \Psi \quad \text { for } \quad A \in \mathfrak{N}
$$

determines a closed, densely defined, anti-linear operator $S$ on $\mathfrak{H}$. Its polar decomposition

$$
S=J \Delta^{1 / 2},
$$

defines the modular operator $\Delta$ and the modular conjugation $J$ of the pair $(\mathfrak{N}, \Psi)$. The fundamental theorem of Tomita and Takesaki states that

$$
J \Psi=\Psi, \quad J \mathfrak{N} J=\mathfrak{N}^{\prime}
$$

and

$$
\Delta^{\imath t} \Psi=\Psi, \quad \Delta^{\imath t} \mathfrak{N} \Delta^{-\imath t}=\mathfrak{N}
$$

for all $t \in \mathbf{R}$.

To make the connection with the quantum dynamical system $\left(\mathfrak{M}, \mathscr{S}^{\beta}, \tau\right)$ note that, according to the previous section, the canonical cyclic representation $(\mathscr{H}, \pi, \Omega)$ associated with $\mathscr{S}^{\beta}$ is faithful. Thus $\Omega$ is a separating cyclic vector for $\pi(\mathfrak{M})$, and we can apply the above construction to the pair $(\pi(\mathfrak{M}), \Omega)$. By a slight abuse of language, we shall say that the operators $J$ and $\Delta$ obtained in this way are the modular conjugation and modular operator of the system $\left(\mathfrak{M}, \mathscr{S}^{\beta}, \tau\right)$. It follows from Tomita-Takesaki's theorem that the formula

$$
\pi^{\sharp}(A) \equiv J \pi(A) J,
$$

defines an anti-linear representation of $\mathfrak{M}$ on the commutant $\pi(\mathfrak{M})^{\prime}$. We shall see that this dual representation ([AW, HHW]) plays a fundamental role in our problem. Another immediate consequence of Tomita-Takesaki's theorem and of Takesaki's characterization of the modular group $\Delta^{\imath t}$ (Theorem 5.3.10 in [BR2]) is 
Proposition 5.1. Let $\left(\mathfrak{M}, \mathscr{S}^{\beta}, \tau\right)$ be a quantum dynamical system, and $(\mathscr{H}, \pi, \Omega)$ its cyclic representation. Assume that $\mathscr{S}^{\beta}$ is a $(\tau, \beta)-K M S$ state. Then the Liouvillean $L$ of the system is related to its modular operator $\Delta$ by the formula

$$
\Delta=\exp (-\beta L)
$$

The modular structure enjoys very simple covariance properties under unitary transformation (inner automorphisms). In particular the spectrum of the Liouvillean is invariant under such a transformation.

Lemma 5.2. Let $(\mathfrak{M}, \mathscr{S}, \tau)$ be a quantum dynamical system, $(\mathscr{H}, \pi, \Omega)$ its cyclic representation, $L$ its Liouvillean and $J$ its modular conjugation. Furthermore let $V$ be a unitary element of $\mathfrak{M}$, and denote by $\gamma$ the associated inner automorphism of $\mathfrak{M}$, i.e.,

$$
\gamma(A)=V^{*} A V
$$

for any $A \in \mathfrak{M}$. To $V$ we associate the unitary operator

$$
U \equiv \pi(V) \pi^{\sharp}(V),
$$

on $\mathscr{H}$. Then $\left(\mathfrak{M}, \mathscr{P} \circ \gamma, \gamma^{-1} \circ \tau \circ \gamma\right)$ is a quantum dynamical system.

1. Its cyclic representation is given by $(\mathscr{H}, \pi, U \Omega)$.

2. Its Liouvillean is $U L U^{*}$.

3. Its modular conjugation is again $J$.

This is again a simple consequence of Tomita-Takesaki's theorem, and the proof will be omitted. We end this section with a powerful result which describes how the modular structure is altered by a small perturbation of the dynamics. It is an immediate rephrasing of a well known theorem of Araki [AR2] (see also [BR2], Theorem 5.4.4 and the remarks after it).

Theorem 5.3. Let $\left(\mathfrak{M}, \mathscr{S}^{\beta}, \tau\right)$ be a quantum dynamical system, $(\mathscr{H}, \pi, \Omega)$ its cyclic representation, $L$ its Liouvillean, and $J$ its modular conjugation. Assume that $\mathscr{S}^{\beta} i$ is the unique $(\tau, \beta)$-KMS state of $\mathfrak{M}$. Then, for any self-adjoint $V \in \mathfrak{M}$, the formula

$$
\tau_{V}^{t}(A) \equiv \pi^{-1}(\exp (i(L+\pi(V)) t) \pi(A) \exp (-i(L+\pi(V)) t))
$$

defines a $\mathrm{W}^{*}$ - dynamical system on $\mathfrak{M}$. Furthermore,

1. $\Omega \in D(\exp (-\beta(L+\pi(V)) / 2))$, and the vector state $\mathscr{S}_{V}^{\beta}$ associated with

$$
\Omega_{V} \equiv \frac{\exp (-\beta(L+\pi(V)) / 2) \Omega}{\|\exp (-\beta(L+\pi(V)) / 2) \Omega\|},
$$

is the unique $\left(\tau_{V}, \beta\right)-K M S$ state of $\mathfrak{M}$.

2. The cyclic representation of the perturbed system $\left(\mathfrak{M}, \mathscr{S}_{V}^{\beta}, \tau_{V}\right)$ is $\left(\mathscr{H}, \pi, \Omega_{V}\right)$.

3. Its Liouvillean is $L_{V}=L+\pi(V)-\pi^{\sharp}(V)$.

4. Its modular conjugation is again $J$.

We are now fully equipped to proceed with the proof of our main result. 


\section{Proofs of Theorem 3.3 and Theorem 3.4}

As a warm up, let us describe in detail the modular structure of the isolated spin at inverse temperature $\beta$. Recall that the observable algebra is $\mathfrak{M}_{s} \equiv M_{2}$. Since it is a factor (i.e., its center $\mathfrak{M}_{s} \cap \mathfrak{M}_{s}^{\prime}$ is trivial), the Gibbs state $\mathscr{S}_{s}^{\beta}$ given in Eq. (2.1) is the unique KMS state of the system (see [BR2], Theorem 5.3.29). We denote by $\left(\mathscr{H}_{s}, \pi_{s}, \Psi_{s}\right)$ the corresponding canonical cyclic representation of $\mathfrak{M}_{s}$ which, according to the GNS construction, can be realized in the following way: The Hilbert space is

$$
\mathscr{H}_{s} \equiv M_{2}
$$

with the familiar inner product

$$
(X, Y) \equiv \operatorname{Tr}\left(X^{*} Y\right) .
$$

The representant $\pi_{s}(A)$ acts by left multiplication i.e., for any $A \in \mathfrak{M}_{s}$ and $X \in \mathscr{H}_{s}$,

$$
\pi_{s}(A): X \mapsto A X .
$$

The unit vector

$$
\Psi_{s} \equiv \frac{1}{\sqrt{Z_{s}^{\beta}}} \exp \left(-\beta H_{s} / 2\right),
$$

is obviously cyclic and separating for $\pi_{s}\left(\mathfrak{M}_{s}\right)$, and satisfies

$$
\mathscr{S}_{s}^{\beta}(A)=\left(\Psi_{s}, \pi_{s}(A) \Psi_{s}\right) .
$$

Thus there is a unique self-adjoint operator $L_{s}$ on $\mathscr{H}_{s}$ such that

$$
\pi_{s}\left(\exp \left(i t H_{s}\right) A \exp \left(-i t H_{s}\right)\right)=\exp \left(i t L_{s}\right) \pi_{s}(A) \exp \left(-i t L_{s}\right)
$$

for $A \in \mathfrak{M}_{s}$, and

$$
L_{s} \Psi_{s}=0 .
$$

One easily checks that the operator defined by

$$
L_{s}: X \mapsto\left[H_{s}, X\right],
$$

has the required properties, and therefore is the Liouvillean of the system. It follows from Proposition 5.1 that the modular operator of the pair $\left(\pi_{s}\left(\mathfrak{M}_{s}\right), \Omega_{s}\right)$ is given by

$$
\Delta_{s} \equiv \exp \left(-\beta L_{s}\right): X \mapsto \exp \left(-\beta H_{s}\right) X \exp \left(\beta H_{s}\right) .
$$

Going back to the definitions (5.1), (5.2), its modular conjugation is immediately identified as

$$
J_{s}: X \mapsto X^{*},
$$

from which we conclude that

$$
\pi_{s}^{\sharp}(A): X \mapsto X A^{*} .
$$

Along the same line, we shall now describe the modular structure of the isolated boson gas at inverse temperature $\beta$. Recall that the algebra of observables is $\mathfrak{M}_{b} \equiv$ $\pi_{A W}\left(\mathscr{C}_{l o c}\right)^{\prime \prime}$, where $\pi_{A W}$ is the Araki-Woods representation corresponding to the 
equilibrium state $\mathscr{S}_{b}^{\beta}$. By construction, the cyclic representation of the reservoir at thermal equilibrium is $\left(\mathscr{H}_{b}, \pi_{b}, \Psi_{b}\right)$, where

$$
\pi_{b}(A)=A
$$

As in the case of the isolated spin, it is straightforward to identify the Liouvillean as the operator on $\mathscr{H}_{b}$ defined by

$$
L_{b}: X \mapsto\left[H_{b}, X\right]
$$

Note that, in this case, the Liouvillean is identical with the original Hamiltonian $H_{B}$. Proceeding as before we can write down the modular operator of the pair $\left(\pi_{b}\left(\mathfrak{M}_{b}\right), \Psi_{b}\right)$,

$$
\Delta_{b} \equiv \exp \left(-\beta L_{b}\right): X \mapsto \exp \left(-\beta H_{b}\right) X \exp \left(\beta H_{b}\right)
$$

Using Definitions (5.1) and (5.2), we see that the modular conjugation $J_{b}$ is characterized by

$$
\begin{aligned}
J_{b} \exp \left(-\beta H_{b} / 2\right) W(\sqrt{1+\rho} f)|\Omega\rangle\langle\Omega| W(\sqrt{\rho} f) \exp \left(\beta H_{b} / 2\right)= \\
W(\sqrt{1+\rho} f)^{*}|\Omega\rangle\langle\Omega| W(\sqrt{\rho} f)^{*} .
\end{aligned}
$$

Since $\exp \left(-\beta H_{b} / 2\right)=\Gamma(\exp (-\beta \omega / 2))$, a well known property of second quantized contractions (see [SI], Sect. I.4) gives

$$
\exp \left(-\beta H_{b} / 2\right) W(f) \Omega=\exp \left(-\frac{1}{4}\left(f,\left(1-\mathrm{e}^{-\beta \omega}\right) f\right)\right) W(\exp (-\beta \omega / 2) f) \Omega .
$$

If $f \in D(\exp (\beta \omega / 2))$, it follows from this formula that $W(f) \Omega \in D\left(\exp \left(\beta H_{b} / 2\right)\right)$, and

$$
\exp \left(\beta H_{b} / 2\right) W(f) \Omega=\exp \left(\frac{1}{4}\left(f,\left(\mathrm{e}^{\beta \omega}-1\right) f\right)\right) W(\exp (\beta \omega / 2) f) \Omega .
$$

Inserting (6.2) and (6.3) in the above characterization of $J_{b}$ leads to

$$
J_{b} W(\sqrt{\rho} f)|\Omega\rangle\left\langle\Omega\left|W(\sqrt{1+\rho} f)=W(\sqrt{1+\rho} f)^{*}\right| \Omega\right\rangle\langle\Omega| W(\sqrt{\rho} f)^{*},
$$

from which it becomes apparent that

$$
J_{b}: X \mapsto X^{*} .
$$

The dual representation is given by

$$
\pi_{b}^{\sharp}(A): X \mapsto\left(A X^{*}\right)^{*},
$$

in particular

$$
\pi_{b}^{\sharp}\left(\pi_{A W}(W(f))\right): X \mapsto W\left(\rho^{1 / 2} f\right)^{*} X W\left((1+\rho)^{1 / 2} f\right)^{*} .
$$

From this, a simple calculation shows that 


$$
\begin{aligned}
& \pi_{b}\left(\pi_{A W}(W(\sqrt{1+\rho} f))\right) \pi_{b}^{\sharp}\left(\pi_{A W}(W(\sqrt{\rho} f))^{*}\right): X \mapsto W(f) X, \\
& \pi_{b}\left(\pi_{A W}(W(\sqrt{\rho} f))^{*}\right) \pi_{b}^{\sharp}\left(\pi_{A W}(W(\sqrt{1+\rho} f))\right): X \mapsto X W(f),
\end{aligned}
$$

and since $\mathfrak{M}_{b}^{\prime}=\pi_{b}^{\sharp}\left(\mathfrak{M}_{b}\right)$, the irreducibility of the Fock representation allows us to conclude that $\mathfrak{M}_{b} \vee \mathfrak{M}_{b}^{\prime}=\mathscr{D}\left(\mathscr{H}_{b}\right)$. Hence we recover the well known facts that $\mathfrak{M}_{b}$ is a factor and that $\mathscr{S}_{b}^{\beta}$ is the the unique KMS state of the isolated boson gas at inverse temperature $\beta$.

We can now describe the modular structure of the combined spin-boson system. In the absence of interaction i.e., when $\lambda=0$, the state

$$
\mathscr{S}_{0}^{\beta} \equiv \mathscr{S}_{s}^{\beta} \otimes \mathscr{S}_{b}^{\beta},
$$

is the unique $\left(\tau_{0}, \beta\right)$-KMS state on $\mathfrak{M}=\mathfrak{M}_{s} \otimes \mathfrak{M}_{b}$. The quantum dynamical system $\left(\mathfrak{M}, \mathscr{S}_{0}^{\beta}, \tau_{0}\right)$ has a canonical cyclic representation $(\mathscr{H}, \pi, \Psi)$ defined by

$$
\begin{gathered}
\mathscr{H} b=\mathscr{H}_{s} \otimes \mathscr{H} b_{b}, \\
\pi=\pi_{s} \otimes \pi_{b}, \\
\pi^{\sharp}=\pi_{s}^{\sharp} \otimes \pi_{b}^{\sharp}, \\
\Psi=\Psi_{s} \otimes \Psi_{b} .
\end{gathered}
$$

The vector $\Psi$ is cyclic and separating for $\pi(\mathfrak{M})$, and the corresponding modular operator and modular conjugation are given by

$$
\begin{gathered}
\Delta=\Delta_{s} \otimes \Delta_{b}, \\
J=J_{s} \otimes J_{b} .
\end{gathered}
$$

Finally the Liouvillean is

$$
L_{0}=L_{s} \otimes I+I \otimes L_{b} .
$$

To obtain the modular structure of the coupled system, we would like to follow the perturbative approach of Theorem 5.3. This is not directly possible, due to the unboundedness of the coupling term $Q \otimes \varphi_{A W}(\alpha)$. However, the following twist avoids this complication: Define

$$
V_{\lambda} \equiv \exp \left(i \lambda Q \otimes \varphi_{A W}(i \alpha / \omega)\right)
$$

One easily checks that Hypotheses (H1)-(H2) imply $\alpha / \omega \in D\left(\omega^{-1 / 2}\right)$, from which we can conclude that $V_{\lambda} \in \mathfrak{M}$. Let us denote by $\gamma_{\lambda}$ the corresponding inner automorphism of $\mathfrak{M}$. Using Weyl's relation (2.3), an explicit calculation shows that

$$
\tau_{\lambda}^{t}=\gamma_{\lambda}^{-1} \circ \xi_{\lambda}^{t} \circ \gamma_{\lambda}
$$

where

$$
\xi_{\lambda}^{t}(A)=\exp \left(i\left(H_{0}+T_{\lambda}\right) t\right) A \exp \left(-i\left(H_{0}+T_{\lambda}\right) t\right)
$$

and $T_{\lambda}$ is the self-adjoint element of $\mathfrak{M}$ given by

$$
T_{\lambda}=\gamma_{\lambda}\left(\sigma_{z} \otimes I\right)-\sigma_{z} \otimes I
$$

Since $\xi_{0}=\tau_{0}$, we know from the previous paragraphs that $\left(\mathfrak{M}, \mathscr{S}_{0}^{\beta}, \xi_{0}\right)$ is a quantum dynamical system, and that $\mathscr{S}_{0}^{\beta}$ is the unique $\left(\xi_{0}, \beta\right)$-KMS state. One further checks that 


$$
\pi\left(\xi_{\lambda}^{t}(A)\right)=\exp \left(i\left(L_{0}+\pi\left(T_{\lambda}\right)\right) t\right) \pi(A) \exp \left(-i\left(L_{0}+\pi\left(T_{\lambda}\right)\right) t\right)
$$

implements the dynamics in the cyclic representation. At this point we are ready to apply Theorem 5.3, which shows that $\xi_{\lambda}$ defines a quantum dynamical system with unique KMS state a inverse temperature $\beta$. The Liouvillean of this system is given by

$$
\widetilde{L}_{\lambda} \equiv L_{0}+\pi\left(T_{\lambda}\right)-\pi^{\sharp}\left(T_{\lambda}\right) .
$$

Applying now Lemma 5.2 , we conclude that $\tau_{\lambda}$ also has a unique KMS state $\mathscr{S}_{\lambda}^{\beta}$, and that the Liouvillean of $\left(\mathfrak{M}, \mathscr{S}_{\lambda}^{\beta}, \tau_{\lambda}\right)$ is given by

$$
L_{\lambda}=\pi(V) \pi^{\sharp}(V) \widetilde{L}_{\lambda} \pi\left(V^{*}\right) \pi^{\sharp}\left(V^{*}\right) .
$$

This formula can be explicitly evaluated to obtain

$$
L_{\lambda}=L_{0}+\lambda \pi_{s}(Q) \otimes \varphi_{A W}(\alpha)-\lambda \pi_{s}^{\sharp}(Q) \otimes \varphi_{A W}^{\sharp}(\alpha),
$$

where $\varphi_{A W}^{\sharp}$ denotes the field operator of the dual Araki-Woods representation $\pi_{b}^{\sharp}$ i.e.,

$$
\pi_{b}^{\sharp}\left(\pi_{A W}(W(f))\right)=\exp \left(-i \varphi_{A W}^{\sharp}(f)\right) .
$$

For calculational purposes, let us develop a more explicit formula. Using the tensor product realizations $\mathscr{H}_{s}=\mathfrak{H}_{s} \otimes \mathfrak{H}_{s}$ and $\mathscr{H}_{b}=\mathfrak{H}_{b} \otimes \mathfrak{H}_{b}$, we can write

$$
L_{\lambda}=L_{s} \otimes I+I \otimes L_{b}+\lambda(Q \otimes I) \otimes \varphi_{A W}(\alpha)-\lambda(I \otimes Q) \otimes \varphi_{A W}^{\sharp}(\alpha)
$$

with

$$
\begin{aligned}
& L_{s} \equiv H_{s} \otimes I-I \otimes H_{s}, \\
& L_{b} \equiv H_{b} \otimes I-I \otimes H_{b},
\end{aligned}
$$

and

$$
\begin{aligned}
& \varphi_{A W}(\alpha) \equiv \varphi\left((1+\rho)^{1 / 2} \alpha\right) \otimes I+I \otimes \varphi\left(\rho^{1 / 2} \bar{\alpha}\right), \\
& \varphi_{A W}^{\sharp}(\alpha) \equiv \varphi\left(\rho^{1 / 2} \alpha\right) \otimes I+I \otimes \varphi\left((1+\rho)^{1 / 2} \bar{\alpha}\right) .
\end{aligned}
$$

We summarize the above discussion in

Theorem 6.1. Let $(\mathscr{H}, \pi, \Omega)$ be the cyclic representation of the non-interacting spinboson system $\left(\mathfrak{M}, \mathscr{S}_{0}^{\beta}, \tau_{0}\right)$ at inverse temperature $\beta<\infty$. For any $\lambda \in \mathbf{R}$ there exists a cyclic and separating vector $\Psi_{\lambda}^{\beta} \in \mathscr{H}$ such that

$$
\mathscr{S}_{\lambda}^{\beta}(A) \equiv\left(\Psi_{\lambda}^{\beta}, \pi(A) \Psi_{\lambda}^{\beta}\right)
$$

is the unique $\left(\tau_{\lambda}, \beta\right)$-KMS state of $\mathfrak{M}$. Furthermore the Liouvillean of the interacting spin-boson system is given by Eq. (6.4).

Theorem 4.2 reformulates the problem of return to equilibrium as a spectral problem for the operator $L_{\lambda}$. Note that $L_{0}$ has the following spectrum:

$$
\begin{aligned}
\sigma_{a c}\left(L_{0}\right) & =\mathbf{R}, \\
\sigma_{s c}\left(L_{0}\right) & =\emptyset, \\
\sigma_{p p}\left(L_{0}\right) & =\{-2,0,2\} .
\end{aligned}
$$


Clearly, \pm 2 are simple eigenvalues with eigenvectors $\chi \pm \otimes \chi \mp \otimes \Psi_{b}$, while 0 is a twofold degenerate eigenvalue with eigenvectors $\chi \pm \otimes \chi \pm \otimes \Psi_{b}$. When the interaction term is "switched on," one naturally expects all these eigenvalues to "turn into resonances," except for 0 which must remain an eigenvalue. The precise way in which the degenerate eigenvalue 0 splits into resonance and eigenvalue is the content of Einstein's A-B law.

The method developed in [JP1] for the analysis of the spectrum of the operator $H_{\lambda}$ immediately applies to $L_{\lambda}$. The reason is the following: The fundamental tool in [JP1] is a spectral deformation of $H_{\lambda}$. This deformation is induced by a complex translation of the variable $s$ in the representation of $\mathscr{H}_{b}$ as the Fock space over $L^{2}\left(\mathbf{R} \times \mathrm{S}^{2}, d s d \sigma\right)$. In this representation, the interaction term in $H_{\lambda}$ is given by ([JP1], Theorem 3.1 and Eq. 3.6)

$$
H_{I} \equiv Q \otimes \varphi\left(\alpha_{\beta}\right),
$$

where $\varphi$ is the Segal field operator of the corresponding Fock representation of CCR, and

$$
\alpha_{\beta} \equiv\left(\frac{s}{1-\exp (-\beta s)}\right)^{1 / 2} \widetilde{\alpha} \in H^{2}\left(\delta, L^{2}\left(\mathrm{~S}^{2}\right)\right),
$$

as a consequence of Hypothesis (H2). A simple calculation shows that the corresponding term in $L_{\lambda}$ is

$$
L_{I} \equiv(Q \otimes I) \otimes \varphi\left(\alpha_{\beta}\right)-(I \otimes Q) \otimes \varphi\left(\exp (-\beta s / 2) \alpha_{\beta}\right),
$$

which clearly enjoys similar analyticity properties. One can show that a complex translation moves the essential spectrum away from the real axis and reveals the resonances (see Fig. 1). These resonances can be computed by the familiar RayleighSchrödinger series. The radius of convergence of these perturbative expansions is essentially determined by the singularities of the function $\alpha_{\beta}$. Hypothesis (H3) further ensures that, for small $\lambda$, the resonances have a positive width.

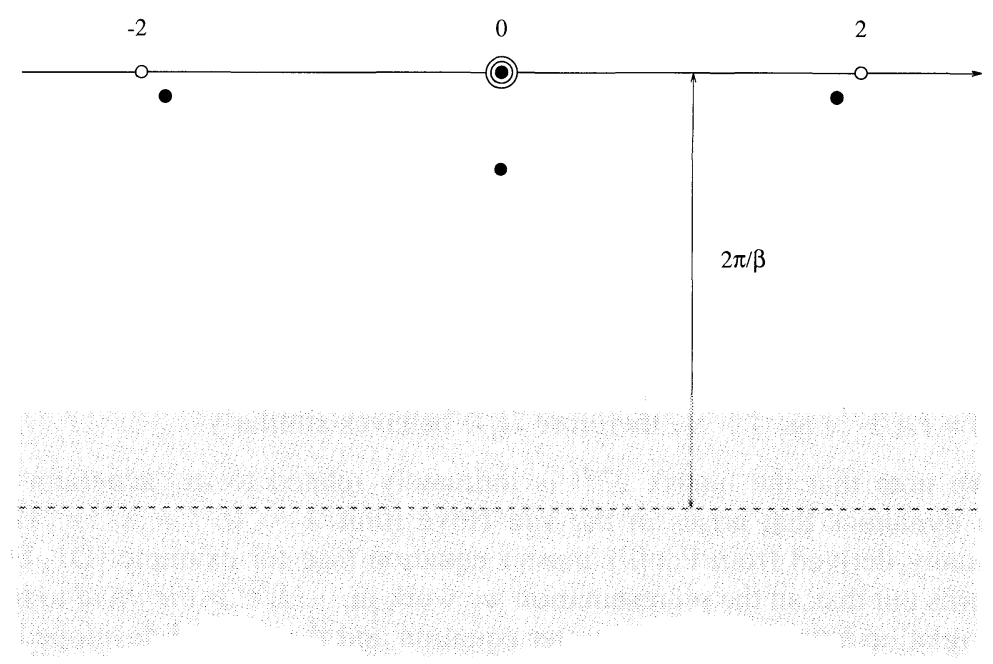

Fig. 1. The eigenvalues of $L_{0}$ (white discs) and the resonances of $L_{\lambda}$ (black discs). The complex translation moves the essential spectrum of the Liouvillean into the shaded area. The first singularity of the function $\alpha_{\beta}$ in the lower half-plane lies on the dotted line 
Recall that $\Gamma_{ \pm}^{\beta}$ and $\Pi_{ \pm}^{\beta}$ were defined in Theorem 3.5. We further set

$$
\begin{gathered}
\Gamma^{\beta} \equiv \Gamma_{-}^{\beta}+\Gamma_{+}^{\beta}, \\
\Pi^{\beta} \equiv \Pi_{+}^{\beta}-\Pi_{-}^{\beta} .
\end{gathered}
$$

Then a simple adaptation of Theorem 2.2 and Proposition 4.7 in [JP1] gives

Theorem 6.2. Suppose that Hypotheses (H1)-(H2) are satisfied. Then there exists a dense subspace $\mathscr{E} \subset \mathscr{H}$ and, for each $\eta \in] 0, \delta[$, a constant $\Lambda(\eta)>0$ such that for $\lambda \in]-\Lambda(\eta), \Lambda(\eta)[$ and $\Phi, \Psi \in \mathscr{E}$, the functions

$$
z \mapsto\left(\Phi,\left(L_{\lambda}-z\right)^{-1} \Psi\right),
$$

have a meromorphic continuation from the upper half plane onto the region

$$
\text { (Q) } \equiv\{z: \operatorname{Im}(z)>-\eta\} \text {. }
$$

The poles of matrix elements (6.6) in (O) are independent of $\Phi$ and $\Psi$. They are identical to the eigenvalues of a quasi-energy operator $\Sigma_{\lambda}$ on $\mathscr{H}_{s}$. This operator is analytic for $|\lambda|<\Lambda(\eta)$, with a power expansion of the form

$$
\Sigma_{\lambda}=L_{s}+\sum_{n=1}^{\infty} \lambda^{2 n} \Sigma^{(2 n)} .
$$

The matrix $\Sigma^{(2)}$ can be explicitly computed and, denoting by $P_{E}$ the eigenprojections of $L_{s}$, we have

$$
P_{ \pm 2} \Sigma^{(2)} P_{ \pm 2}=\left( \pm \Pi^{\beta}-i \Gamma^{\beta}\right),
$$

for the simple eigenvalues, and

$$
P_{0} \Sigma^{(2)} P_{0}=\left(\begin{array}{cc}
-2 i \Gamma_{-}^{\beta} & 2 i \Gamma_{-}^{\beta} \mathrm{e}^{-\beta} \\
2 i \Gamma_{+}^{\beta} \mathrm{e}^{\beta} & -2 i \Gamma_{+}^{\beta}
\end{array}\right),
$$

for the degenerate one. Note that the eigenvalues of the matrix (6.8) are 0 and $-4 i \Gamma^{\beta}$.

An immediate consequence of the above result and of Proposition 4.1 in [CFKS] is that there is a constant $\ell(\beta)>0$ such that for $0<|\lambda|<\ell(\beta)$ the spectrum of $L_{\lambda}$ is purely absolutely continuous, except for the simple eigenvalue 0 . The proof of Theorem 2.3 is completed by invoking Theorem 4.2 and Corollary 4.3.

Remark 1. It follows from the the proofs of Theorem 4.6 and Proposition 4.7 in [JP1] that $\Lambda(\eta)=O\left(\beta^{-1}\right)$ as $\beta \uparrow \infty$, therefore $\ell(\beta)$ behaves similarly.

Remark 2. We note that the matrix $\Sigma^{(2)}$ is intimately related to the generator of the Markovian dynamics that arises in the van Hove limit $\lambda \rightarrow 0, t=\lambda^{-2} \tau$. This generator is usually derived from Pauli's master equation (see for example [D1, D2, $\mathrm{D} 3$ or $\mathrm{M}]$ ). It turns out that, in the representation we work in, $-i \Sigma^{(2)}$ is identical to this generator. The relation between Pauli's master equation and the method developed in this paper will be discussed in more detail in [JP2].

We now sketch the proof of Theorem 3.4. We will only consider the limit $t \uparrow+\infty$, a similar argument can be used for $t \downarrow-\infty$. We invoke the dynamical consequence of Theorem 6.2 i.e., Theorem 2.5 in [JP1]. 
Theorem 6.3. Suppose that Hypotheses (H1)-(H2) are satisfied. Then there exists a dense subspace $\mathscr{E} \subset \mathscr{H}$ and, for each $\eta \in] 0, \delta[$, a constant $\Lambda(\eta)>0$ with the following property: For $|\lambda|<\Lambda(\eta)$ there are two maps $W_{\lambda}^{ \pm}: \mathscr{E} \rightarrow \mathscr{H}_{s}$ such that for any $\Phi, \Psi \in \mathscr{E}$, one has $\left(W_{\lambda}^{-} \Phi, W_{\lambda}^{+} \Psi\right)=(\Phi, \Psi)$ and

$$
\left(\Phi, \exp \left(-i t L_{\lambda}\right) \Psi\right)=\left(W_{\lambda}^{-} \Phi, \exp \left(-i \Sigma_{\lambda} t\right) W_{\lambda}^{+} \Psi\right)+O(\exp (-\eta t))
$$

as $t \rightarrow+\infty$.

If $U(\theta)$ denote the group of translations introduced in Sect. 4 of [JP1], then $\mathscr{E}$ can be chosen to be the set of vectors which are analytic for $U(\theta)$ in a strip $\mathfrak{S}(\delta)$. In particular, we can choose $\mathscr{E}$ independently of $\beta$.

We define the set of states $\mathscr{M}_{0}$ and the algebra $\mathfrak{M}_{0}$ as follows. Let

$$
\mathscr{C}_{0} \equiv\left\{X \otimes \pi_{A W}(W(f)): \tilde{f} \in H^{2}\left(\delta, L^{2}\left(\mathrm{~S}^{2}\right)\right)\right\}
$$

denote by $\widetilde{\mathcal{N}_{0}}$ the set of vector states associated with vectors in $\pi^{\sharp}\left(\mathscr{B}_{0}\right) \Psi_{\lambda}^{\beta}$ and set $\widetilde{\mathfrak{M}}_{0}=\pi\left(\mathscr{C}_{0}\right)$. We define $\mathscr{N}_{0}$ as the set of finite convex linear combinations of states in $\widetilde{\mathscr{N}_{0}}$ and $\mathfrak{M}_{0}$ as the linear span of $\widetilde{\mathfrak{M}}_{0}$. Clearly $\mathscr{N}_{0}$ and $\mathfrak{M}_{0}$ enjoy the properties stated in Theorem 3.4. Note also that it is sufficient to prove (3.4) for $\mathscr{S} \in \widetilde{\mathcal{N}_{0}}$ and $A \in \widetilde{\mathfrak{M}}_{0}$. Let $\gamma(\lambda)$ be the negative imaginary part of the complex eigenvalue of $\Sigma_{\lambda}$ closest to the real axis. Then it clearly satisfies the properties stated in the theorem. By Eq. (4.3) and Theorem 6.3 the proof of Theorem 3.4 reduces to showing that the vectors $\pi(A) \Psi_{\lambda}^{\beta}$ and $\pi^{\sharp}(A) \Psi_{\lambda}^{\beta}$ belong to the set $\mathscr{E}$ for $A \in \mathscr{C}_{0}$. In the representation of $\mathscr{H}_{b}$ as the Fock space over $L^{2}\left(\mathbf{R} \times \mathrm{S}^{2}, d s d \sigma\right)$ used in [JP1], we have that

$$
\begin{aligned}
& \pi\left(X \otimes \pi_{A W}(W(f))\right)=\pi_{s}(X) \otimes W\left(\left(\frac{s}{1-\exp (-\beta s)}\right)^{1 / 2} \tilde{f}\right), \\
& \pi^{\sharp}\left(X \otimes \pi_{A W}(W(f))\right)=\pi_{s}^{\sharp}(X) \otimes W\left(\left(\frac{s}{\exp (\beta s)-1}\right)^{1 / 2} \tilde{f}\right) .
\end{aligned}
$$

Therefore, from our assumption on $\tilde{f}$ in Definition (6.9), Theorem 3.4 will follow from $\Psi_{\lambda}^{\beta} \in \mathscr{E}$. Using the notation of [JP1], this can be established as follows. Let $L_{\lambda}(-i \theta)$ be the deformed Liouvillean defined as in Sect. 4 of [JP1]. By Theorem 4.6 in [JP1], there is a constant $\Lambda>0$, so that for $\frac{|\lambda|}{\Lambda}<\theta<\delta$ and $\Phi, \Phi^{\prime} \in \mathscr{E}$, one has

$$
\left(\Phi, \Psi_{\lambda}^{\beta}\right)\left(\Psi_{\lambda}^{\beta}, \Phi^{\prime}\right)=\left(U(i \theta) \Phi, Q_{\lambda}(-i \theta) U(-i \theta) \Phi^{\prime}\right),
$$

where $Q_{\lambda}(-i \theta)$ is the spectral projection of the deformed Liouvillean $L_{\lambda}(-i \theta)$ corresponding to the eigenvalue zero. Let now $\Phi=U(-i \theta) \Phi_{0}$ and $\Phi^{\prime}=U(i \theta) \Phi_{0}$ with $\Phi_{0}$ an analytic vector for $U(\theta)$ in the strip $\mathfrak{S}(2 \delta)$. Then $\Phi, \Phi^{\prime} \in \mathscr{E}$, and from Eq. (6.10) we conclude that

$$
\left|\left(U(-i \theta) \Phi_{0}, \Psi_{\lambda}^{\beta}\right)\right|\left|\left(\Psi_{\lambda}^{\beta}, U(i \theta) \Phi_{0}\right)\right|=\left|\left(\Phi_{0}, Q_{\lambda}(-i \theta) \Phi_{0}\right)\right| \leq C_{\theta, \lambda}\left\|\Phi_{0}\right\|^{2} .
$$

Thus for $\frac{|\lambda|}{\Lambda}<\theta<\delta$ we have

$$
\Psi_{\lambda}^{\beta} \in \mathrm{D}(U(-i \theta)) \cap \mathrm{D}(U(i \theta)),
$$


and therefore $U(\theta) \Psi_{\lambda}^{\beta}$ is analytic in $\mathfrak{S}(\delta)$. All the necessary estimates follow from Theorem 4.6 in [JP1]. This completes the proof of Theorem 3.4.

\section{References}

[A1] Arai, A.: On a model of a harmonic oscillator coupled to a quantized, mass-less, scalar field, I. J. Math. Phys. 22, 2539 (1981)

[A2] Arai, A.: On a model of a harmonic oscillator coupled to a quantized, mass-less, scalar field, II. J. Math. Phys. 22, 2549 (1981)

[AM] Amann, A.: Ground states of a spin-boson model. Ann. Phys. 208, 414 (1991)

[AR1] Araki, H.: Positive cone, Radon-Nikodym theorems, relative Hamiltonian and the Gibbs condition in statistical mechanics. An application of the Tomita-Takesaki theory. In: $\mathrm{C}^{*}-$ Algebras and their Applications to Statistical Mechanics and Quantum Field Theory. Kastler, D., (ed.) Amsterdam: North-Holland, 1976

[AR2] Araki, H.: Relative Hamiltonian for faithful normal states of a von Neumann algebra. Publ. R.I.M.S. 9, 165 (1973)

[AW1] Araki, H., Woods, E.J.: Representation of the canonical commutation relations describing a non relativistic infinite free Bose gas. J. Math. Phys. 4, 637 (1963)

[BR1] Bratteli, O., Robinson, D.: Operator Algebras and Quantum Statistical Mechanics I. Berlin, Heidelberg, New York: Springer, 1979

[BR2] Bratteli, O., Robinson, D.: Operator Algebras and Quantum Statistical Mechanics II. Berlin, Heidelberg, New York: Springer, 1981

[BFS] Bach, V., Fröhlich, J., Sigal, I.M.: Mathematical theory of non-relativistic matter and radiation. Preprint

[CA] Cannon, J.T.: Infinite volume limits of the canonical free Bose gas states on the Weyl algebra. Commun. Math. Phys. 29, 89 (1973)

[CDG] Cohen-Tannoudji, C., Dupont-Roc, J., Grynberg, G.: Photons and Atoms - Introduction to Quantum Electrodynamics. Berlin, Heidelberg, New York: Springer, 1991

[CFKS] Cycon, H., Froese, R., Kirsch, W., Simon, B.: Schrödinger Operators. Berlin, Heidelberg, New York: Springer, 1987

[CFS] Cornfeld, I.P., Fomin, S.V., Sinai, Y.G.: Ergodic Theory. Berlin, Heidelberg, New York: Springer, 1982

[CH] Chaiken, J.M.: Number operators for representations of the canonical commutation relations. Commun. Math. Phys. 8, 164 (1968)

[D1] Davies, E.B.: Markovian master equations. Commun. Math. Phys. 39, 91 (1974)

[D2] Davies, E.B.: Markovian master equations II. Math. Ann. 219, 147 (1976)

[D3] Davies, E.B.: Quantum Theory of Open Systems. New York: Academic Press, 1976

[DI] Dirac, P.A.M.: The quantum theory of the emission and absorption of radiation. Proc. Royal Soc. London, Series A 114, 243 (1927)

[E] Einstein, A.: Zur Quantentheorie der Strahlung. Physik. Zeitschr. 18, 121 (1917)

[FNV1] Fannes, M., Nachtergaele, B., Verbeure, A.: The equilibrium states of the spin-boson model. Commun. Math. Phys. 114, 537 (1988)

[FNV2] Fannes, M., Nachtergaele, B., Verbeure, A.: Quantum tunneling in the spin-boson model. Europhysics Lett. 4, 963 (1987)

[FNV3] Fannes, M., Nachtergaele, B., Verbeure, A.: Tunneling in the equilibrium state of a spin-boson model. J. Phys. A 21, 1759 (1988)

[GJ] Glimm, J., Jaffe, A.: Quantum field theory models. In: Statistical Mechanics and Quantum Field Theory. DeWitt, C., Stora, R., (eds.) New York, London, Paris: Gordon and Breach, 1970

[HA] Haag, R.: Local Quantum Physics. Berlin, Heidelberg, New York: Springer, 1993

[HE] Heitler, W.: The Quantum Theory of Radiation. Oxford: Oxford University Press, 1954

[HHW] Haag, R., Hugenholtz, N.M., Winnink, M.: On the equilibrium states in quantum statistical mechanics. Commun. Math. Phys. 5, 215 (1967)

[HS1] Hübner, M., Spohn, H.: Radiative decay: Non perturbative approaches. Preprint

[HS2] Hübner, M., Spohn, H.: Spectral properties of the spin-boson Hamiltonian. Preprint

[JP1] Jakšić, V., Pillet, C.-A.: On a model for quantum friction II. Fermi's golden rule and dynamics at positive temperature. Commun. Math. Phys. 
[JP2] Jakšić, V., Pillet, C.-A.: On a model for quantum friction IV. Return to equilibrium, (in preparation)

[LCD] Legget, A.J. et al.: Dynamics of the dissipative two-state system. Rev. Mod. Phys. 59, 1 (1987)

[LP] Lewis, J.T., Pulè, J.V.: The equilibrium states of the free boson gas. Commun. Math. Phys. 36, 1 (1974)

[MA] Maassen, H.: Return to thermal equilibrium by the solution of a quantum Langevin equation. J. Stat. Phys. 34, 239 (1984)

[M] Martin, Ph.A.: Modèles en Mécanique Statistique des Processus Irréversibles (Lectures Notes in Physics, 103). Berlin, Heidelberg, New York: Springer, 1979

[P] Pauli, W.: Pauli Lectures on Physics: Volume 4. Statistical Mechanics. Edited by C.P. Enz. Cambridge, MA: The MIT Press, 1973

[PA] Pais, A.: "Subtle is the Lord...", The Science and the Life of Albert Einstein. Oxford, New York: Oxford University Press, 1982

[PU] Pulè, J.V.: The Bloch equations. Commun. Math. Phys. 38, 241 (1974)

[RO1] Robinson, D.W.: C*- algebras and quantum statistical mechanics. In: C*- Algebras and their Applications to Statistical Mechanics and Quantum Field Theory. Kastler, D., (ed.) Amsterdam: North-Holland, 1976

[RO2] Robinson, D.W.: Return to equilibrium. Commun. Math. Phys. 31, 171 (1973)

[RS] Reed, M., Simon, B.: Methods of Modern Mathematical Physics III. Scattering Theory. London: Academic Press, 1978

[SA] Sakai, S.: $C^{*}-$ Algebras and $W^{*}$ - Algebras. Berlin, Heidelberg, New York: Springer, 1971

[SD] Spohn, H., Dümcke, R.: Quantum tunneling with dissipation and the Ising model over R. J. Stat. Phys. 41, 381 (1985)

[SDLL] Smedt, P., Dürr, D., Lebowitz, J.L., Liverani, C.: Quantum system in contact with a thermal environment: Rigorous treatment of a simple model. Commun. Math. Phys. 120, 120 (1988)

[SI] Simon, B.: The $P(\varphi)_{2}$ Euclidean (Quantum) Field Theory. Princeton NJ: Princeton University Press, 1974

[SC] Schwinger, J.: Selected Papers on Quantum Electrodynamics. New York: Dover, 1958

[SP] Spohn, H.: Ground state(s) of the spin-boson Hamiltonian. Commun. Math. Phys. 123, 277 (1989)

[WW] Weisskopf, V., Wigner, E.P.: Berechnung der natürlichen Linienbreite auf Grund der Diracschen Lichtheorie. Z. Phys. 63, 54 (1930)

Communicated by B. Simon

This article was processed by the author using the Springer-Verlag TEX PJour1g macro package 1991. 
